Archives de sciences sociales des religions

180 | octobre-décembre 2017

Bulletin bibliographique

\title{
Le monastère : une utopie en acte
}

Jean-Louis Fabiani

\section{OpenEdition}

Journals

Édition électronique

URL : https://journals.openedition.org/assr/29739

DOI : 10.4000/assr.29739

ISSN : $1777-5825$

Éditeur

Éditions de l'EHESS

Édition imprimée

Date de publication : 1 décembre 2017

Pagination : 239-248

ISSN : 0335-5985

\section{Référence électronique}

Jean-Louis Fabiani, «Le monastère : une utopie en acte », Archives de sciences sociales des religions [En ligne], 180 | octobre-décembre 2017, mis en ligne le 01 décembre 2017, consulté le 22 septembre 2021. URL : http://journals.openedition.org/assr/29739 ; DOI : https://doi.org/10.4000/assr.29739 


\title{
Le temps des moines
}

\author{
À propos de: \\ Hervieu-LÉger Danièle, Le temps des moines. Clôture et hospitalité, \\ Paris, Presses universitaires de France, 2017, 712 p.
}

\section{Jean-Louis Fabiani \\ Une utopie en acte}

Quel est le temps des moines dont le livre traite? Un temps relativement court au regard de l'histoire, et plutôt long pour un sociologue: de la fin de la première moitié du XIX ${ }^{\mathrm{e}}$ siècle à nos jours, depuis la (re)fondation post-révolutionnaire jusqu'aux crises d'un âge post-chrétien, comme le nomme l'auteure ou, à tout le moins, d'un christianisme éclaté, selon Michel de Certeau. L'enquête de Danièle Hervieu-Léger est admirable et extrêmement bien documentée. On peut lire le livre sans faire de pause, dans la continuité que permet une écriture limpide, débarrassée de tout jargon sociologique et associée à une maîtrise époustouflante de l'objet. Un livre de maturité qui réconcilie avec la sociologie, pour qui pourrait ressentir quelques doutes à l'égard de l'utilité de cette discipline. Les moines dont il est question sont pour une part hors du temps social: l'utopie monastique dont ils sont porteurs les conduit à s'affranchir des contraintes que les temporalités sociales suscitent, particulièrement à l'âge moderne et à l'âge du capitalisme. La Règle affranchit les moines de ces contraintes pour imposer d'autres formes de scansion du temps, d'autres rythmes qui s'opposent en tout temps, quelle que soit la forme que prend la temporalité sociale, aux usages purement sociaux du calendrier. La clôture manifeste la discordance entre le temps social ordinaire et le temps monastique. La clôture n'est pourtant jamais totale. La porosité est la règle, bien qu'elle varie selon les temps et les ordres. Du monastère on entend le grondement du monde, et peut-être avec une meilleure acoustique qu'ailleurs, car on l'entend sans les bruits parasites qui pullulent dans l'univers ordinaire. Le monachisme moderne s'est constitué dans des conditions historiques qu'on peut dire défavorables, puisque le processus de sécularisation inscrit dans la longue durée en fait une des manifestations de l'anti-modernité par excellence, d'une forme de réaction aux tendances profondes de la transformation sociale engendrée par les mutations de l'économie. Les gestes refondateurs, en particulier ceux de Muard et de Guéranger à La Pierre-qui-Vire et à Solesmes, constituent 
une réponse différée à la destruction révolutionnaire, et constituent une éclaircie plutôt brève en France, puisque la Troisième République met à nouveau à son programme l'affaiblissement de l'institution religieuse. La fin des années 1960 voit surgir une crise de type nouveau, au moment où quelques monastères embrassent l'effervescence sociale du temps pour offrir d'autres perspectives refondatrices (à Boquen et à Maredsous notamment) et d'autres devenir les bastions d'une position anti-conciliaire. Même si les chiffres que donne l'auteure conduisent à penser à un déclin de l'institution monastique, dramatique en certains lieux comme l'illustrent le vieillissement de la population et le tarissement des vocations, le phénomène ne présente pas de caractère linéaire; il existe des formes de "réveil » et d'histoires à succès, y compris dans la période récente. Il s'agit donc d'une histoire complexe, qui épouse l'histoire chahutée du catholicisme moderne, mais qui a sa temporalité et ses convulsions propres en dépit du caractère centralisé de l'institution catholique, laquelle autorise des trajectoires monastiques bien plus diverses qu'on ne pourrait le penser. Le livre permet de mieux comprendre l'entrelacement de diverses temporalités: dans des communautés le plus souvent de petite taille, la gestion du temps repose d'abord sur le temps biologique des abbés et des moines (il s'agit ici d'une histoire en personnes, passionnante): s'y superpose le temps des monastères, celui de l'Église et celui de la société dans son ensemble. Le fil conducteur de l'enquête est la notion d'utopie: le monastère est une utopie en acte, expression d'un geste radical de fondation (la plus part du temps exprimé en "refondation" mais qui apparaît souvent comme une invention de tradition fondée sur un retour à la tradition) et sur le charisme, au sens à la fois théologique et sociologique, qui ne sont pas complètement superposables, on le sait. On constate la continuité de la radicalité chrétienne et donc présupposition d'un temps continu depuis les origines du christianisme, dans la discontinuité, et quelquefois la dissonance des charismes fondateurs. Les grandes tentatives de refondation sont toujours ambivalentes: elles s'inscrivent dans l'histoire de leur temps tout en revendiquant la solidité d'un lignage; à ce titre elles ne sont jamais complètement anti-modernes, y compris les plus réactives. La Règle peut s'exprimer contre le siècle, mais elle est toujours prise dans le siècle.

La qualité de l'enquête est pour une bonne part due au fait que l'auteure prend au sérieux l'utopie monastique, qu'elle la met au cœur de l'investigation. Dans le geste même de leur retrait du monde, les moines portent témoignage de la réalité du Royaume à venir. Il n'est pas possible de parler du temps des moines sans faire mention du temps eschatologique, qui se superpose an temps sociétal et au temps ecclésial, sans que jamais la concordance de ces temps ne soit donnée, sauf peutêtre dans la réussite absolue de la temporalité monastique. L'enquête se trouve déployée de l'intérieur du catholicisme sans jamais transiger avec les réquisitions de la sociologie historique. Un de mes amis anthropologues me disait un jour que la condition de la sociologie de la religion avait pour préalable la sortie de la religion, comme la sociologie de la philosophie présupposerait qu'elle soit historiquement finie. Ce livre prouve le contraire, sans aucune ostentation.

De ce fait, le monastère n'est jamais analysé comme «institution totale» au sens de Goffman, ce qu'il est aussi d'ailleurs et d'une manière expressive puisque la communauté est à elle-même sa propre fin, bien que les aspects "sociétaux » de la vie monastique ne soient jamais passés sous silence. Le renoncement à la 
vie sociale qu'implique la radicalité de l'engagement n'efface pas magiquement les habitus sociaux pas plus que la stratification sociale, comme en témoigne l'importance relative de l'aristocratie au sein de la population des abbés ainsi que la division des tâches dans l'espace du monastère. Les fonctions de contrôle et de surveillance mutuels apparaissent clairement, de même que certaines formes de servitude volontaire qui sont des conditions de l'utopie et qui se manifestent sous des formes historiquement très variables, pour ce qui est du rapport au corps: on note un continuum qui va de la mortification (plutôt rare) au souci de la vie bonne écologique en passant par l'ascèse.

La vie monastique est analysée à partir de la notion weberienne de double éthique: les moines sont des virtuoses dont on exige d'autres formes de manifestation et d'approfondissement de la foi que les fidèles ordinaires, dans la mesure où ils témoignent, de manière utopique, de l'avènement possible du Royaume, à partir d'un travail sur soi qui s'exprime dans les termes de la Règle. Comment cette double éthique peut-elle survivre à l'ère de l'émancipation individuelle où l'approfondissement de soi est devenu une norme universelle? J'emprunte librement cette notion à Pierre-Michel Menger ${ }^{1}$ qui l'a développée à propos de la profession de comédien; j'ai tenté de l'appliquer de manière plus étendue à l'ensemble des formes de présentation de soi dans les sociétés contemporaines ${ }^{2}$, où chacun est requis de devenir un "performeur ${ }^{3}$. Dans la double éthique, il y a un double rapport à la virtuosité. Les fidèles ordinaires peuvent être rangés du côté des spectateurs. La virtuosité est réservée à ceux qui s'engagent à plein temps dans la vie chrétienne, le charisme de l'abbé définissant d'ailleurs un niveau plus élevé de performance dans la performance. Qu'en est-il de la virtuosité performante à l'âge du culte de la performance? La question permet de mieux comprendre l'évolution récente de la vie monastique. D'un côté, ce qui s'est joué à Boquen et à Maredsous, c'est la mise en question de la ligne de partage entre virtuose et spectateur, notamment dans le premier cas à travers l'instauration d'un nouveau type d'espace collectif nommé la Communion où les laïcs peuvent à leur tour devenir des performeurs alors que la notion de virtuosité est elle-même mise en crise. Mais l'on a aussi assisté à l'émergence d'un néo-traditionalisme spectaculaire, comme au Barroux, marqué par la réactivation d'une virtuosité extrême, qui est, comme le remarque l'auteure, très proche de ce qu'on exige dans le monde séculier de l'individu performant. La question de la participation des fidèles, pouvant inclure des fidèles d'autres religions comme dans l'utopie œcuménique, Taizé occupant une place significative dans le livre, est centrale dans l'âge post-conciliaire. Les transformations de la liturgie, qui font l'objet d'une analyse serrée, sont au cœur de la question. L'accès du plus grand nombre à la performance monastique, à travers le passage au français ou la transformation de la configuration spatiale de l'église, rejoint de nombreux débats, fort séculiers mais dont on pourrait tracer les rapports avec des formes de rituels ou de liturgie, à propos de la représentation théâtrale: Jean Vilar envisageait de transformer le spectateur en participant, mais

1. P. M. Menger, La profession de comédien. Formation, activités et carrières dans la démultiplication de soi, Paris, La Documentation française, 1997.

2. J.-L. Fabiani, "O que resta de agente social?", Tempo social, vol 14, n 1, 2002, p. 33-65.

3. A. Ehrenberg, Le culte de la performance, Paris, Calmann-Lévy, 1991. 
il est mort désespéré quelques mois après que les spectateurs d'Avignon montèrent sur le plateau de la cour du lycée Mistral pour échanger avec les acteurs du Living Theatre de Julian Beck et Judith Malina. L'auteure fait d'ailleurs un rapprochement avec Avignon, bref mais intéressant. Où situer la ligne aujourd'hui ? L'enquête montre que c'est une question centrale. Car la clôture n'est jamais totale: elle est affectée par les nouvelles formes de l'hospitalité et notamment par l'atténuation de la différence entre les genres, mais aussi par les contraintes que pose le vieillissement de la population des moines et la nécessité subséquente de la présente constante de soins infirmiers dans l'espace de la clôture. Certains monastères ont un âge moyen supérieur à 80 ans et comptent de nombreux grabataires.

Le paradoxe contemporain est celui du succès des néo-traditionnalistes sur fond de déclin, semble-t-il inexorable, de la vocation. Les «tradis » ont intensifié le spectacle en une époque de «marchandisation» des cultes et des rituels, comme on peut le voir à travers la présentation spectaculaire des rituels de nombreuses religions exotiques par l'ethno-scénographie. Ils offrent des réponses alors qu'une des caractéristiques de l'approfondissement de soi que permet le retrait du monde est précisément le questionnement. On peut y voir une contradiction permanente de la vie monastique, la Règle apportant les réponses à travers une discipline du corps alors que le questionnement demeure et est peut-être intensifié par la prière.

C'est à ce point que l'on peut s'interroger sur l'unité de la forme de vie que propose la vie monastique, qui semble osciller entre l'effort constant pour se dépouiller de son enveloppe corporelle et mondaine d'un côté et le souci de la vie bonne de l'autre. L'ouverture au monde post-conciliaire a renforcé cette «tension essentielle» pour parler comme Thomas Kuhn ${ }^{4}$. Celle-ci est intensifiée par les règles de l'hospitalité, variables, mais qui font aussi l'unité et la cohérence de cette forme de vie. À l'inconnu qui frappe à la porte on ne demande pas son identité, fût-ce au prix de quelques problèmes de maintien de l'ordre. On se souvient que Michel Houellebecq, arrivant à la tombée du jour à la porterie de Ligugé, fut pris pour un SDF. On ne le refoula pas pour autant. Le livre examine la question en se référant au célèbre texte de Derrida sur la question de l'hospitalité: il est incontestable qu'elle constitue aujourd'hui un des plus importants ressorts de la survie de l'utopie monastique, ou à tout le moins de son hétérotopie: la capacité de se constituer en contre société dans un univers où les frontières deviennent beaucoup plus étanches que la clôture du monastère, assumant une fonction de radicalité critique fort différente de la restauration virtuose du néo-traditionalisme et de la patrimonialisation spectaculaire.

Jean-Louis FABIANI EHESS

jean-louis.fabiani@ehess.fr

4. T. Kuhn, La tension essentielle. Tradition et changement dans les sciences, Paris, Gallimard, 1990. 